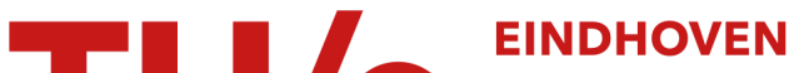 \\ UNIVERSITY OF \\ TECHNOLOGY
}

\section{Interaction of dipolar vortices with a step-like topography}

Citation for published version (APA):

Tenreiro, M., Zavala Sansón, L., \& Heijst, van, G. J. F. (2006). Interaction of dipolar vortices with a step-like topography. Physics of Fluids, 18(5), 056603-1/12. https://doi.org/10.1063/1.2204070

DOI:

$10.1063 / 1.2204070$

Document status and date:

Published: 01/01/2006

Document Version:

Publisher's PDF, also known as Version of Record (includes final page, issue and volume numbers)

Please check the document version of this publication:

- A submitted manuscript is the version of the article upon submission and before peer-review. There can be important differences between the submitted version and the official published version of record. People interested in the research are advised to contact the author for the final version of the publication, or visit the $\mathrm{DOI}$ to the publisher's website.

- The final author version and the galley proof are versions of the publication after peer review.

- The final published version features the final layout of the paper including the volume, issue and page numbers.

Link to publication

\section{General rights}

Copyright and moral rights for the publications made accessible in the public portal are retained by the authors and/or other copyright owners and it is a condition of accessing publications that users recognise and abide by the legal requirements associated with these rights.

- Users may download and print one copy of any publication from the public portal for the purpose of private study or research.

- You may not further distribute the material or use it for any profit-making activity or commercial gain

- You may freely distribute the URL identifying the publication in the public portal.

If the publication is distributed under the terms of Article $25 \mathrm{fa}$ of the Dutch Copyright Act, indicated by the "Taverne" license above, please follow below link for the End User Agreement:

www.tue.nl/taverne

Take down policy

If you believe that this document breaches copyright please contact us at:

openaccess@tue.nl

providing details and we will investigate your claim. 


\title{
Interaction of dipolar vortices with a step-like topography
}

\author{
M. Tenreiro and L. Zavala Sansón \\ Department of Physical Oceanography, CICESE, Km 107 Carretera Tijuana-Ensenada, \\ 22860 Ensenada, Baja California, México \\ G. J. F. van Heijst \\ Fluid Dynamics Laboratory, Eindhoven University of Technology, P.O. Box 513, 5600 MB, \\ Eindhoven, The Netherlands
}

(Received 16 January 2006; accepted 3 April 2006; published online 22 May 2006)

\begin{abstract}
The interaction of a barotropic, dipolar vortex with a step-like topography is studied by means of laboratory experiments in a rotating tank and by numerical simulations based on a quasi-two-dimensional model. Two main configurations are analyzed: when the dipole approaches a "low" or a "high" step with respect to the maximum water depth. For relatively low steps, the vortex crosses the topography with a deflected trajectory, while maintaining its dipolar structure. The sense of this deflection depends on whether the dipole reaches a step-up or a step-down. For high steps, in contrast, the dipole is not able to cross the topography, and the reflection of one of the dipole structures is observed. In both cases, one observes a weak flow along the topography with shallow water on its right. The essential features of the flow evolution for low and high steps can be explained by using arguments of potential vorticity conservation (due to the weakness of viscous effects). In order to determine whether a barotropic dipole is able to cross the step or is reflected, qualitative criteria based on the step height and dipole strength are derived using inviscid arguments. (C) 2006 American Institute of Physics. [DOI: 10.1063/1.2204070]
\end{abstract}

\section{INTRODUCTION}

The interaction of vortices with other vortices, coastal boundaries, and different bottom topographies in a rotating system are important in many areas of geophysical interest. These interactions might be responsible for several meterological, oceanographical, or biological phenomena at different spatial and temporal scales. In particular, mesoscale vortices and currents are often studied by means of laboratory experiments in a rotating tank. ${ }^{1}$ For the case of flows in the presence of lateral boundaries, strong vorticity is created and complex flow structures arise. ${ }^{2,3}$ At the bottom boundary, topographic effects play a crucial role in the flow evolution. These effects are associated either with the bottom Ekman layer, inducing the so-called Ekman friction, and/or with topographic variations, which produce stretching of fluid columns outside the boundary layer. ${ }^{4}$

A basic feature of the flows studied here is their twodimensional (2D) character. Two-dimensional motion is observed in laboratory experiments by using a rotating table, with its axis aligned in the vertical direction (antiparallel with gravity). In this case, a homogeneous fluid moves perpendicular to the rotation axis in a columnar fashion, according to the Taylor-Proudman theorem. When using variable topography there are three-dimensional effects that break the 2D character of the flow. However, for a rotating fluid system with low to moderate Rossby number (which indicates the importance of rotation effects) and low Ekman number, the flow still exhibits a columnar motion, but now modulated by depth changes, which in turn affect the column's relative vorticity. This behavior is here referred to as quasi-twodimensional.
This paper addresses the interaction of dipolar structures with a step-like topography. The objective is to understand the dynamics of this type of vortices when they reach the topography normally, either from deep to shallow water or vice versa. In order to gain a better understanding of the problem, the study is carried out by means of laboratory experiments in a rotating tank and by numerical simulations. The laboratory experiments provide a physical evidence of typical interactions of dipolar vortices with a topographic step. The numerical simulations are based on a barotropic, quasi-two-dimensional model including topography variations.

A step topography has been used in other experimental studies to analyze the evolution of barotropic currents flowing across an underwater escarpment. ${ }^{5-7}$ In particular, Zavala Sansón et al. ${ }^{7}$ showed that the exchange of fluid between shallow and deep regions induce the creation of vortices at the step. An and $\mathrm{McDonald}^{8}$ reported similar results by means of contour dynamics numerical simulations. Another important feature of the coastal current-step problem is the formation of a weak flow along the topography, which moves with shallow water on its right (see also Carnevale et al. ${ }^{9}$ ). In another study, Zavala Sansón et al. ${ }^{10}$ investigated experimentally and numerically the interaction of barotropic monopolar vortices drifting on a topographic $\beta$ plane towards a step-like topography. Their results show that the interaction strongly depends on the step height and on the sign of the vortex: cyclonic (anticyclonic) vortices are able to cross a step-up (-down), whereas anticyclones (cyclones) are "reflected" from this topography. Similar results have been reported in other theoretical and numerical papers. ${ }^{11,12}$

Most of these processes are involved in the present ex- 


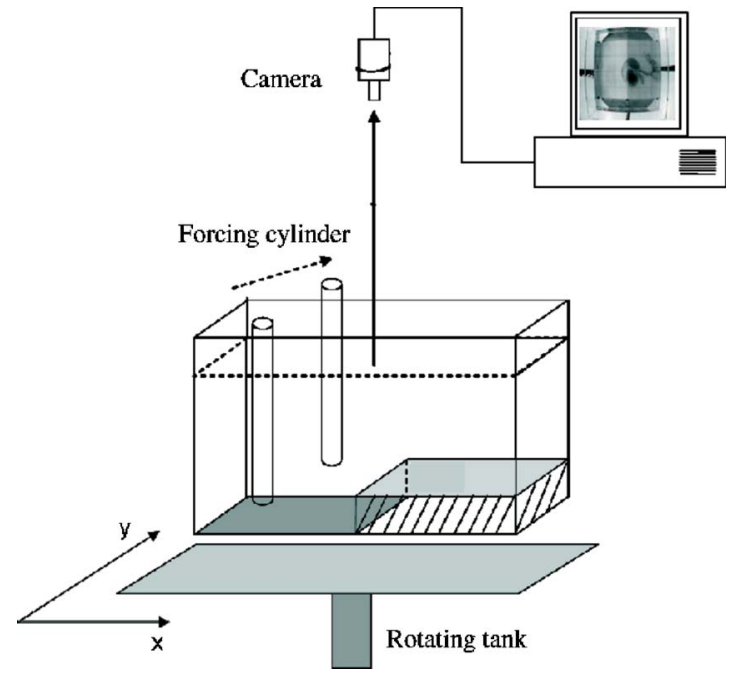

FIG. 1. Schematic view of the experimental setup and the forcing method of dipolar vortices.

periments with dipolar vortices. A crucial difference is, of course, the self-propagation of dipoles towards a step-up or a step-down. The results show that the dipole behavior depends crucially on the step height, and that the quasi-2D barotropic model used to simulate the process represents well the laboratory experiments. Another important result is the formation of a current alongside the step, and the creation of new vortices associated with the dipole-step interaction. Experiments and simulations for relatively low and high steps (compared with the maximum water depth) are presented in Secs. III and IV, respectively. The numerical results are used to determine the critical step height, under which the dipole crosses the topography (with a deflected trajectory), and above which part of the dipole is reflected from the step (Sec. V).

\section{EXPERIMENTAL AND NUMERICAL METHODS}

\section{A. Laboratory}

The laboratory experiments are performed in a rectangular, rotating tank $\left(L_{x} \times L_{y}=150 \mathrm{~cm} \times 100 \mathrm{~cm}\right)$ filled with fresh water. The height of the water column at the deep part of the domain is $H_{0}=20 \mathrm{~cm}$, and two step heights are used: 1 and $5 \mathrm{~cm}$ (Fig. 1). Hereafter these configurations are referred to as low and high steps, respectively, since they crucially determine the flow evolution in two very different ways.

The rotation rate of the tank around the $z$ axis was fixed at $\Omega=0.5 \mathrm{rad} \mathrm{s}^{-1}$, which corresponds to a Coriolis parameter $f=2 \Omega=1 \mathrm{~s}^{-1}$. A typical experiment has a duration of about $200 \mathrm{~s}$. The decay induced by bottom friction is associated with the Ekman period, $T_{E} \equiv H_{0} /(\nu \Omega)^{1 / 2} \approx 280 \mathrm{~s}$, for $\nu=0.01 \mathrm{~cm}^{2} \mathrm{~s}^{-1}$ (kinematic viscosity of the water at $20^{\circ} \mathrm{C}$ ). This time scale is much longer than the rotation period, $T=2 \pi / \Omega \approx 12$, so that the vortices are only slightly affected by the Ekman decay. ${ }^{13}$ The experimental procedure consisted of setting the tank in rotation at the specified constant angular speed about 30 min before the start of an experiment, in order to ensure that the fluid has reached a state of solidbody rotation. The changes in depth due to the step
(1 to $5 \mathrm{~cm}$ ) are more important than the effects of the parabolic free surface (1 to $3 \mathrm{~mm}$ ) so that the latter are ignored. This assertion is supported by numerical simulations, which do not include free surface effects, showing the same tendency as the experiments.

Dipolar vortices are produced by horizontally moving a hollow cylinder $(8 \mathrm{~cm}$ diameter) through the tank center and pulling it out while maintaining its vertical axis parallel with the rotation axis. ${ }^{14}$ The vorticity is generated at the walls of the cylinder and it rapidly organizes into a dipolar structure. Initially, the mean speed of the dipole is $U \simeq 0.6 \mathrm{~cm} \mathrm{~s}^{-1}$ along the $x$ axis. The characteristic vorticity scale $\omega_{0}$ is about $0.5 \mathrm{~s}^{-1}$, so that the Rossby number $\mathrm{Ro}=\omega_{0} / f$ is always smaller than 1, ensuring the two-dimensional character of the flow.

The vortices are visualized by adding fluorescent dye into the core of the vortices for qualitative experiments. In addition, quantitative experiments are performed by using a number of passive tracers $\sim 250 \mu \mathrm{m}$ ) floating on the free surface. The subsequent evolution was recorded with a corotating camera mounted at some distance above the tank. Two different methods were used for quantitative experiments: particle image velocimetry and particle tracking velocimetry. Note that the quantified experiments are independent of the qualitative, dye experiments. The main variations are associated with the forcing method, which originates some differences on the initial velocity and trajectory of the dipole. Nevertheless, the main results were clearly reproducible in all experiments.

\section{B. Simulations}

The numerical simulations are based on a quasi-2D, barotropic model in the $\omega-\psi$ formulation with Ekman friction: ${ }^{4}$

$$
\begin{aligned}
& \frac{\partial \omega}{\partial t}+J(q, \psi)-\frac{\delta_{E}}{2 h} \nabla \psi \cdot \nabla q=\nu \nabla^{2} \omega-\frac{\delta_{E}}{2 h} \omega(\omega+f), \\
& \omega=-\frac{1}{h} \nabla^{2} \psi+\frac{1}{h^{2}} \nabla h \cdot \nabla \psi+\frac{\delta_{E}}{2 h} \frac{2}{h^{2}} J(h, \psi) .
\end{aligned}
$$

Here, $\omega=\partial v / \partial x-\partial u / \partial y$ is the relative vorticity, with $(u, v)$ the horizontal, depth-independent velocity; the potential vorticity is defined as $q=(\omega+f) / h$, where $h(x, y)$ is the fluid depth, $\psi$ is a stream function, $\delta_{E}=(2 \nu / f)^{1 / 2}$ is the thickness of the bottom Ekman layer, and $J$ the Jacobian operator. Note that the local depth $h$ is time independent, according with the rigid lid approximation, and therefore depends only on the spatial shape of the topography. The model includes linear and nonlinear Ekman friction terms (proportional to $\delta_{E}$ ), which provide a good representation of bottom friction effects, always present in the laboratory experiments. Since the duration of a typical simulation is shorter than the Ekman period, similar results can be achieved by using only the classical linear term $\delta_{E} f \omega / 2 h \approx E^{1 / 2} f \omega / 2$, with $E=\delta_{E} / H_{0}$ the Ekman number, and neglecting nonlinear frictional effects. The model [(1) and (2)] is solved by means of a finite differences code. ${ }^{4}$ The domain is a $100 \mathrm{~cm} \times 100 \mathrm{~cm}$ square with no-slip boundary conditions, which is discretized by 
TABLE I. Characteristic parameters of the numerical simulations.

\begin{tabular}{lll} 
Maximum depth & $H_{0}$ & $20 \mathrm{~cm}$ \\
Kinematic viscosity & $\nu$ & $0.01 \mathrm{~cm}^{2} \mathrm{~s}^{-1}$ \\
Rotation period & $T=2 \pi / \Omega$ & $280 \mathrm{~s}$ \\
Dipole atmosphere & $a$ & $8 \mathrm{~cm}$ \\
Initial dipole speed & $U_{0}$ & $0.6 \mathrm{~cm} \mathrm{~s}^{-1}$ \\
& $U_{0}$ (in Fig. 16) & $0.2,0.4,0.6,0.8 \mathrm{~cm} \mathrm{~s}^{-1}$ \\
Step height & $\Delta h$ & low (high) $1(5) \mathrm{cm}$ \\
& $\Delta h$ (in Fig. 16) & $1,2,3,5 \mathrm{~cm}$ \\
\hline \hline
\end{tabular}

$129 \times 129$ grid points. As a consequence, the step-like topography in the simulations is actually a very narrow slope, whose width is much smaller than the vortex size. The timestep is $\Delta t=0.1 \mathrm{~s}$ for all simulations.

The initial condition in all simulations is the ChaplyginLamb dipole. ${ }^{15}$ Using polar coordinates in a reference frame co-moving with the dipole at a constant speed $U_{0}$, the vorticity distribution inside a circle of radius $a$ (the vortex atmosphere) is given by

$$
\omega(r, \theta)=-\frac{2 U_{0} k}{J_{0}(k a)} J_{1}(k r) \sin \theta, \quad 0 \leqslant r \leqslant a,
$$

where $J_{0}$ and $J_{1}$ are the zero- and first-order Bessel functions of the first kind, respectively, and $k$ is a constant such that $a k=3.8317 .{ }^{16}$ According to (3), the peak vorticity of the dipole is $\omega_{0}=-2 U_{0} k / J_{0}(k a) \max \left\{J_{1}(k r)\right\} \approx 11.1 U_{0} / a$. In all simulations, the radius of the vortex atmosphere is $a=8 \mathrm{~cm}$. Analogously to the experiments, the dipole moves along the $x$ axis, starting $30 \mathrm{~cm}$ away from the step. The dipole decay is induced by vorticity diffusion, radial expansion and bottom friction. ${ }^{13}$ Table I shows the numerical parameters used for the simulations, which are representative of the laboratory experiments.

\section{DIPOLE CROSSING A LOW STEP}

In order to understand the effect of the topography on the dipole behavior, the trajectories and structural changes resulting from the interaction with a relatively low step are investigated. In this case, the step height $(1 \mathrm{~cm})$ is small compared with the water depth $(20 \mathrm{~cm})$. The evolution of the dipole is radically different from the case in which a higher step is used, as shall be shown in next section.

\section{A. Laboratory experiments}

Figure 2 shows a sequence of photographs where the dipole approaches the step from the deep part of the domain (left to right). Hereafter, the left (right) side of the domain corresponds with the deep (shallow) region in all figures. The vertical line indicates the position of the step. Initially, the dipole follows approximately a perpendicular trajectory towards the step $(t=30-60 \mathrm{~s})$. When reaching the topographic feature $(t=90 \mathrm{~s})$, the trajectory is deflected in anticyclonic direction. This deflection is maintained during the rest of the experiment $(t=120-180 \mathrm{~s})$. Note that the vortex maintains its dipolar structure during the whole experiment. During the last $30 \mathrm{~s}$, the dipole motion is very slow due to viscous effects. The dipole behavior shown in this figure was systematically observed in several experiments, so that this result can be considered robust.

The change of the dipole trajectory can be explained in terms of potential vorticity conservation per fluid column (considering that viscous effects are very weak):

$$
\frac{\omega+f}{h}=\text { const }
$$

Since $f=2 \Omega$ is constant, variations of the column depth $h$ induce changes in its relative vorticity $\omega$ to maintain the potential vorticity constant. The vorticity columns are squeezed as the dipole crosses the step, which implies the decrease of relative vorticity. As a consequence, the cyclone loses intensity while the anticyclone becomes stronger. Due to this asymmetry induced by the topography the dipole acquires a curved trajectory in the direction of the stronger structure, that is, the anticyclone.

Figure 3 presents, for the same experimental parameters, the case where the dipole reaches the step from the shallow part of the tank (right to left). At the beginning the dipole approaches the step perpendicularly $(t=30-60 \mathrm{~s})$ as in previous case. Once it crosses the topography ( $t=90 \mathrm{~s})$, the trajectory is deflected again, but now in the cyclonic direction.
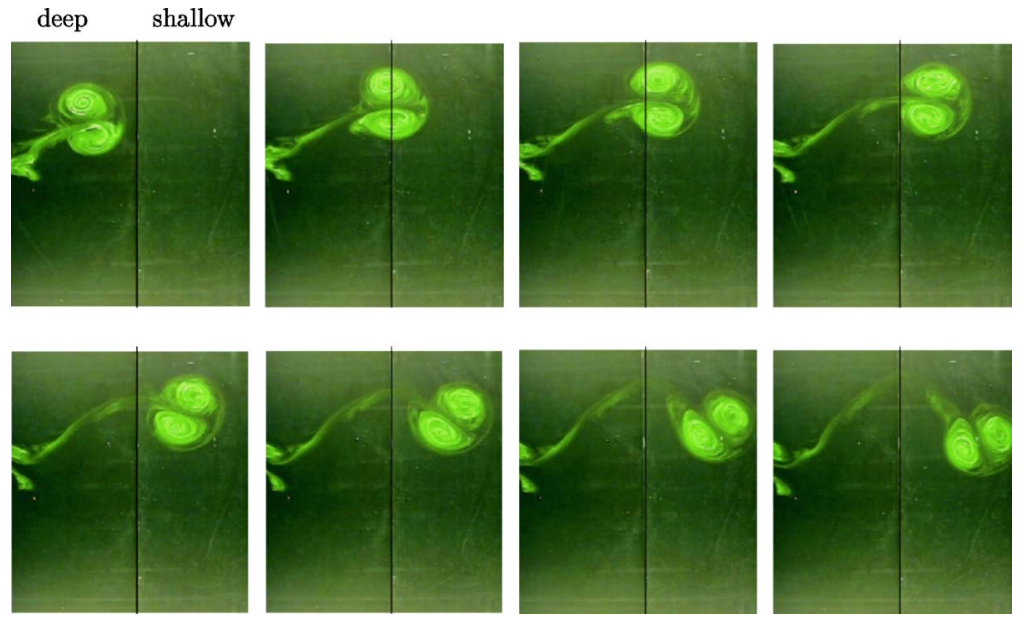

FIG. 2. Top view photographs showing the evolution of an experimental dipole crossing a low step $(1 \mathrm{~cm})$ from deep to shallow water during a time span of $\sim 18$ rotation periods (time interval is $30 \mathrm{~s}$ ). The vortex is visualized with dye inside an area of $\sim 75 \mathrm{~cm} \times 75 \mathrm{~cm}$. 

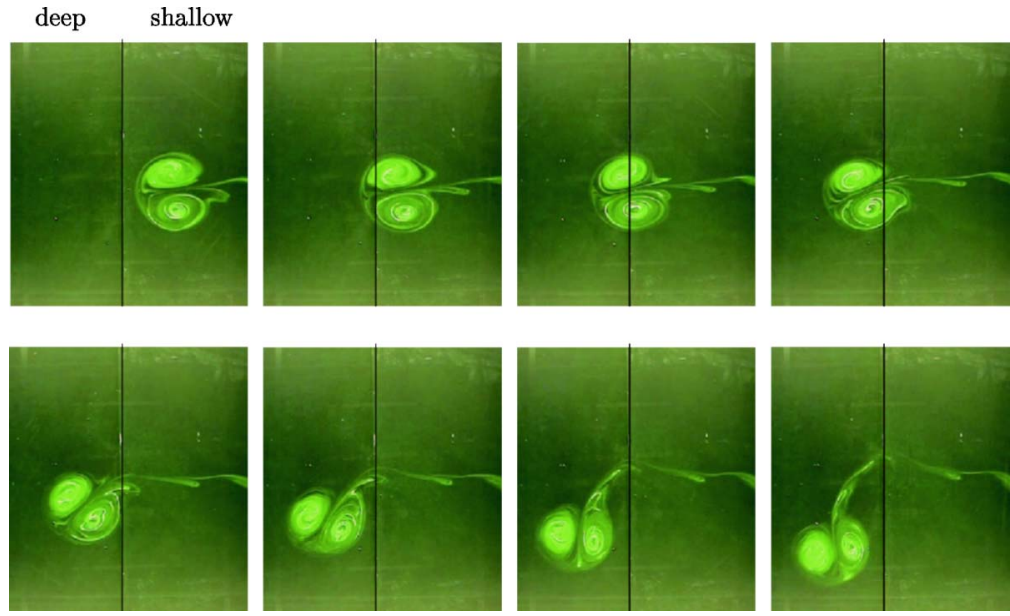

FIG. 3. Top view photographs showing the evolution of an experimental dipole crossing a low step $(1 \mathrm{~cm})$ from shallow to deep water during a time span of $\sim 18$ rotation periods (time interval is $30 \mathrm{~s}$ ). The vortex is visualized with dye inside an area of $\sim 75 \mathrm{~cm} \times 75 \mathrm{~cm}$.
In this case, the vorticity columns are stretched due to the depth increase and therefore the relative vorticity increases too. Thus, the anticyclone gets weaker, while the cyclone is intensified. As a result, the trajectory is deviated in cyclonic direction.

Typical trajectories of experimental dipoles crossing either from deep to shallow or vice versa are shown in Fig. 4. It must be remarked that this behavior was observed in several experiments. Of course, the measured trajectories were not identical because the dipole generation method implies inevitable variations on its direction and initial velocity. However, the curved trajectories of the experimental dipoles are consistent with the intensification of one of its parts by stretching or squeezing effects when crossing the topography.

\section{B. Numerical simulations}

The principal objective of the numerical simulations is to show that the quasi-2D barotropic model given by Eqs. (1) and (2) is able to reproduce the laboratory experiments, which allows one to invoke the physical mechanisms previously described. Furthermore, the simulations permit to study with great detail some of the complex processes occurring near the step, which are difficult to observe in the experiments.

Figure 5 (upper panels) shows the evolution of relative vorticity contours from a simulation of a dipole moving from deep to shallow water. It can be observed that the vorticity distribution is affected by the presence of the step at early stages of the simulation $(t=30 \mathrm{~s})$. When the dipole gets close to the step, negative vorticity is created near the topography (ahead of the vortex) due to squeezing effects, since water is pushed towards the shallow region. As a consequence, a return flow from shallow to deep water at both sides of the dipole is produced, which is responsible for the creation of positive vorticity by stretching effects at the deep side of the step. Once the dipole crosses the topography, the fluid columns are squeezed and the cyclonic part becomes weaker than the anticyclonic one. Therefore, the dipole trajectory is deflected in the direction of the anticyclonic part due to the loss of symmetry. It must be noted that, because of the curved trajectory, a large cell of positive vorticity is created at the deep part next to the anticyclone $(t=90-150 \mathrm{~s})$. This new vorticity distribution originated along the step interacts with the dipole at later times $(t=150-210 \mathrm{~s})$. Another important feature to be observed is the relative vorticity being accumulated at the upper boundary of the domain, which is maintained during the whole simulation. This is caused by a weak current alongside the step with shallow water on its right, which eventually interacts with the upper boundary.

The squeezing and stretching mechanisms responsible for the creation of vorticity along the step are evident in Fig. 5 (lower panels), which correspond to the evolution of passive tracers advected by the flow. A group of 1000 tracers
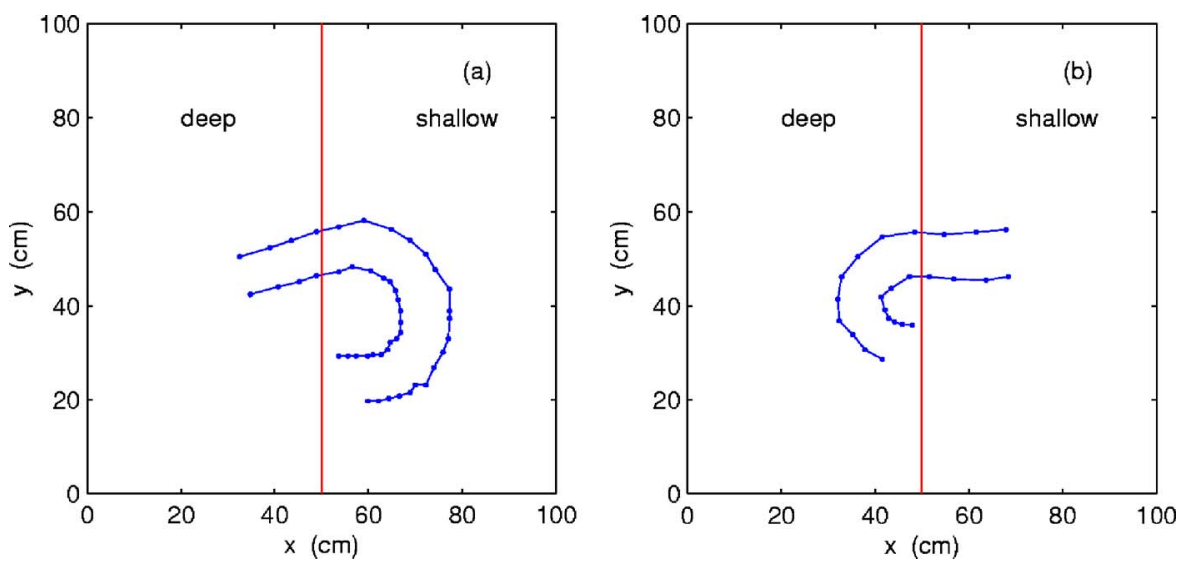

FIG. 4. Typical experimental trajectories of dipolar vortices crossing a low step from (a) deep to shallow, and (b) shallow to deep. The initial dipole speed is approximately $0.6 \mathrm{~cm} \mathrm{~s}^{-1}$. The position of the cyclonic and anticyclonic parts, indicated every $10 \mathrm{~s}$ (dots), are directly measured from video recordings. 

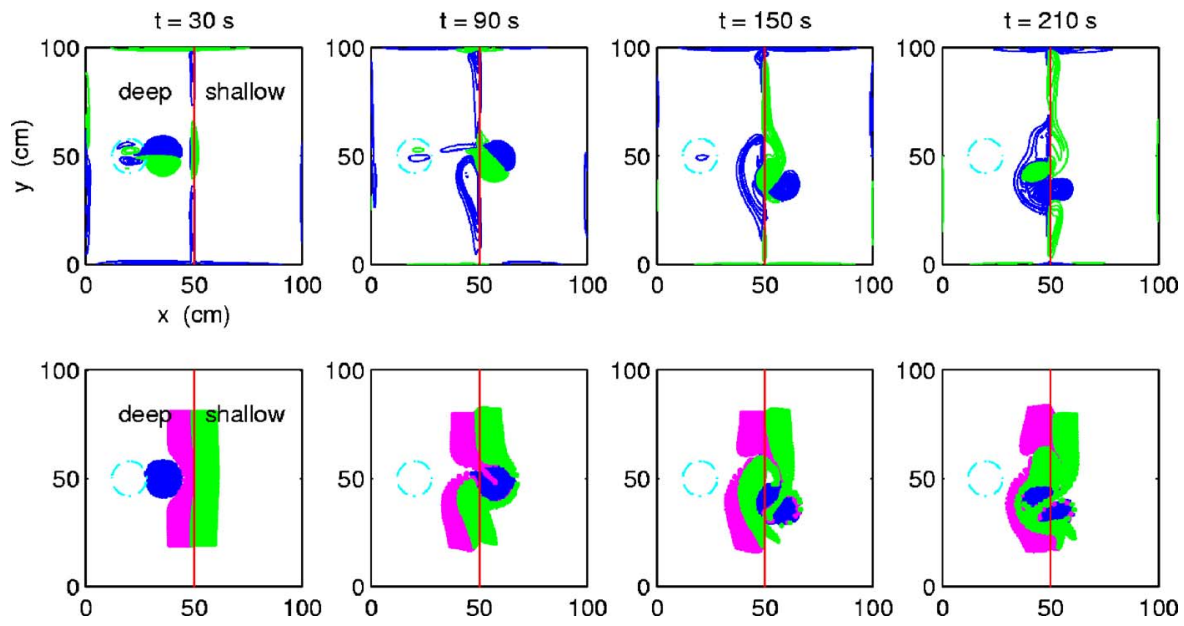

FIG. 5. Upper row: Numerically calculated vorticity contours of a dipole crossing a low step $(1 \mathrm{~cm})$ from shallow to deep water. Black (gray) contours indicate positive (negative) vorticity values. The contour interval is $0.01 \mathrm{~s}^{-1}$, and the maximum/minimum vorticity is $1 /-1 \mathrm{~s}^{-1}$. The dipole parameters are $\left(a=8, U_{0}=0.6 \mathrm{~cm} \mathrm{~s}^{-1}\right)$. The initial atmosphere is indicated by the dashed circle. Lower row: Evolution of passive tracers initially arranged inside three areas: (a) 1000 dark particles at a circle with radius $8 \mathrm{~cm}$ and centered with the dipole's atmosphere, (b) 1000 gray particles inside a $10 \mathrm{~cm} \times 60 \mathrm{~cm}$ at the deep region, and (c) 1000 bright particles inside a $10 \mathrm{~cm} \times 60 \mathrm{~cm}$ rectangle at the shallow side. were randomly distributed inside the initial dipole atmosphere, and two rectangular areas of 1000 particles were set at both sides of the step. By comparing with upper panels, note that the displacement of particles from deep to shallow water corresponds with the creation of negative vorticity and vice versa. For example, some particles initially at the shallow part of the domain are pushed towards the deep region by the influence of the deflected dipole trajectory, thus creating the cyclonic vorticity cell observed at $t=90-150 \mathrm{~s}$.

When the dipole approaches the step from the shallow side the behavior of the flow is very similar to the previous case, but now with vorticity signs exchanged. This is shown in the corresponding sequence of vorticity contours in Fig. 6 (upper panels). The dipole is stretched when crossing the step, resulting an intensification of the cyclone and therefore a deflected trajectory towards this structure. Analogously, also, the curved trajectory of the dipole pushes fluid from deep to shallow, thus generating a negative vorticity cell $(t$ $=90-150 \mathrm{~s}$ ). Figure 6 (lower panels) shows the corresponding evolution of tracers. There are, however, some minor differences when the dipole approaches a step-up or -down, as can be appreciated from a closer inspection of Figs. 5 and 6. The reason for such differences is that the shallow water model (1) is not invariant under transformations of both vorticity and step sign. These symmetry considerations are further discussed in last section. On the other hand, a common result in both cases is that the dipole interaction with the step gives rise to a flow along the topography towards the upper part of the domain, with shallow water to the right.

In order to compare with the laboratory experiments, Fig. 7(a) shows the positions of the absolute peak vorticity centers of the cyclonic and anticyclonic structures of a numerical and an experimental dipole crossing towards the deep region. The initial position of the numerical vortex, $\left(x_{0}, y_{0}\right)=(78 \mathrm{~cm}, 50 \mathrm{~cm})$, was set to match approximately the measured position of the experimental dipole. After crossing the step, the trajectories are almost circular as the vortex motion is damped by viscous effects before moving back to the step. It can be noticed that the numerical dipole covers a somewhat shorter trajectory. This is due to a slight underestimation of the dipole speed at initial stages, as can be observed from Fig. 7(b). Nevertheless, there is a reasonable quantitative agreement during several rotation periods. This provides confidence on the numerical scheme for a systematic study with different flow parameters, as shall be shown in the last section.
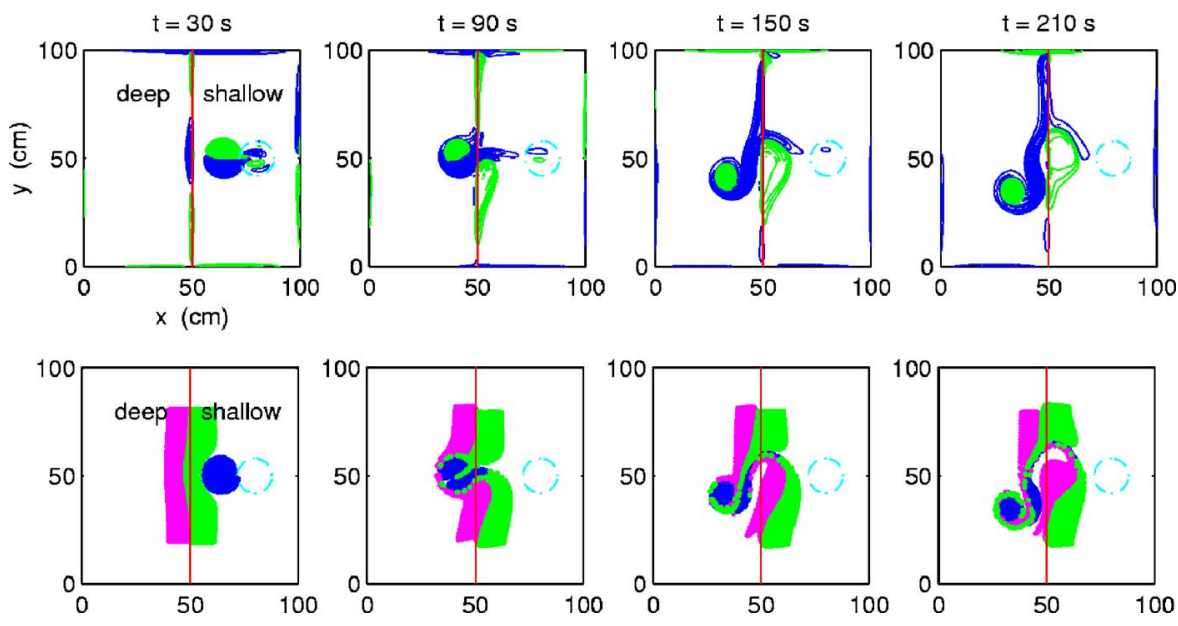

FIG. 6. Upper row: Vorticity contours of a dipole crossing a low step $(1 \mathrm{~cm})$ from deep to shallow water. Lower row: Evolution of passive tracers. Vorticity contours, dipole parameters and initial arrangement of tracers as in Fig. 5. 

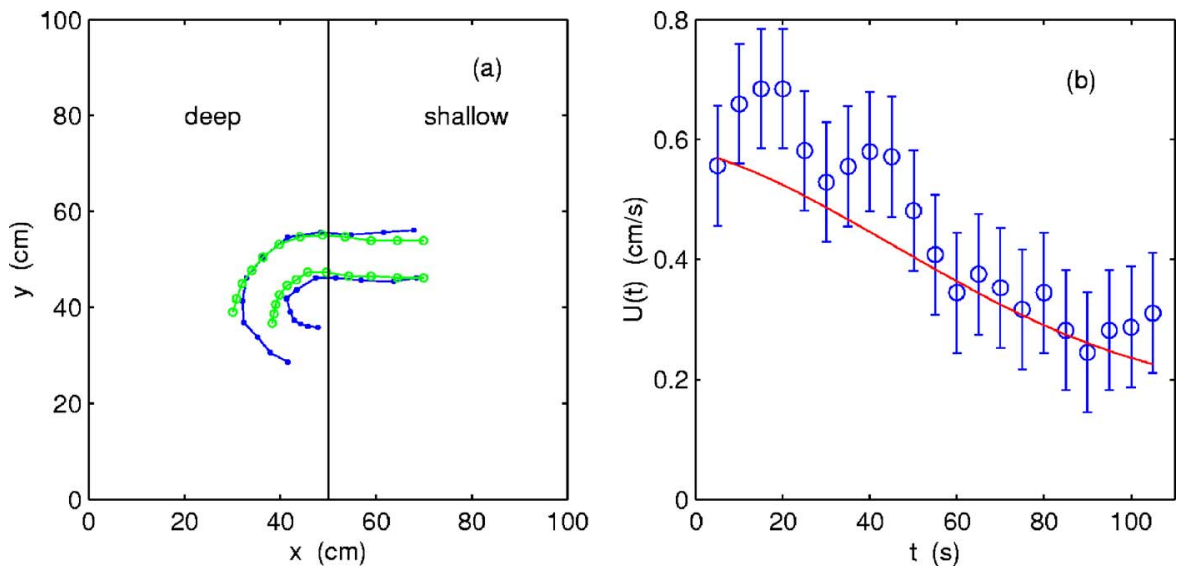

FIG. 7. (a) Typical trajectory of an experimental dipole (black line) crossing from shallow to deep compared with the calculated trajectory in a numerical simulation (gray line) during $110 \mathrm{~s}$. The position of the cyclonic and anticyclonic parts are indicated every $10 \mathrm{~s}$ (dots and circles). (b) Dipole speed obtained from the experimental dipole (denoted by circles with error bars) and from the numerical simulation (solid line).

\section{Point vortices}

In order to show the inviscid character of the curved trajectory after experiencing the step influence, a point vortex model is used. This formulation is analogous to the model described by Velasco Fuentes and van Heijst ${ }^{14}$ for dipolar vortices on a $\beta$ plane. To introduce the effect of the topography, it is necessary to associate an area $A$ to each singular vortex, such that its circulation is given by

$$
\Gamma=\omega A,
$$

with $\omega$ the relative vorticity. Thus, changes in depth $H$ of the column due to topographic effects have to be compensated by changes in $A$, in order to conserve volume

$$
V=A(x, y) H(x, y)=A_{0} H_{0}=\text { const, }
$$

where $A_{0}$ and $H_{0}$ are the area and depth of reference, respectively. In addition, the potential vorticity conservation is

$$
\frac{\omega_{0}+f}{H_{0}}=\frac{\omega+f}{H} .
$$

Using (6) and (7) in Eq. (5), the expression for the circulation of a point vortex modulated by topography is given by

$$
\Gamma(x, y)=\Gamma_{0}+f A_{0}\left[1-\frac{H_{0}}{H(x, y)}\right],
$$

where $\Gamma_{0}$ is the initial circulation.

Before experiencing changes in depth, a dipole can be represented by two point vortices with oppositely signed circulation: $\Gamma_{0}$ and $-\Gamma_{0}\left(\right.$ let $\left.\Gamma_{0}>0\right)$. Under their mutual influence, the dipolar vortex moves with speed

$$
U=\frac{\Gamma_{0}}{2 \pi r}
$$

where $r$ is the (constant) separation of the vortices. Considering topographic variations, the circulation of the cyclonic and anticyclonic parts are given by

$$
\Gamma^{+}=\Gamma_{0}+f A_{0}\left[1-\frac{H_{0}}{H}\right]
$$

$$
\Gamma^{-}=-\Gamma_{0}+f A_{0}\left[1-\frac{H_{0}}{H}\right]
$$

Once the circulation changes, the speed of the vortices is given by

$$
\begin{gathered}
U^{+}=\frac{\left|\Gamma^{-}\right|}{2 \pi r}, \\
U^{-}=\frac{\left|\Gamma^{+}\right|}{2 \pi r}
\end{gathered}
$$

(note that the speed of each vortex depends on the circulation of the contiguous one).

Consider the case of the dipole propagating from deep to shallow water. Due to the asymmetry induced by the change of depth, the vortices move along circular trajectories, with the separation between them constant. Evidently, the stronger, anticyclonic vortex moves along the inner circle with a lower speed than the cyclone (see Fig. 8). Since the angular velocity of the vortices around the circular trajectory is the same:

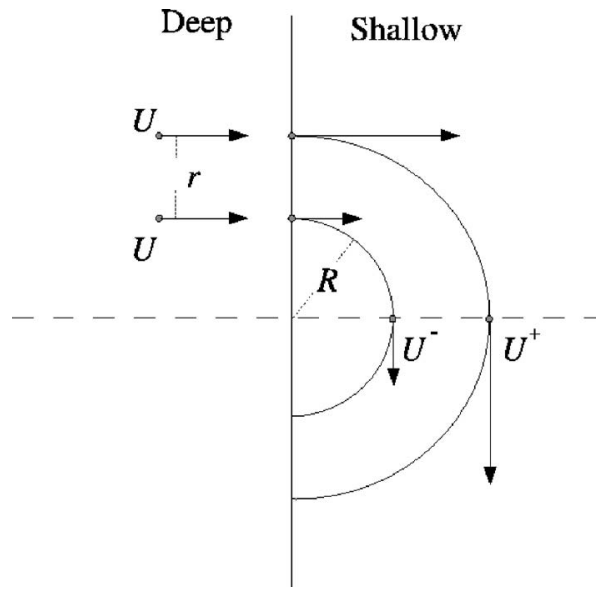

FIG. 8. Schematic view of the trajectory of a point dipolar vortex modulated by topography when crossing from deep to shallow water. The anticyclone is intensified while the cyclone gets weaker. The dipole trajectory is deflected towards the intensified, anticyclonic part. 


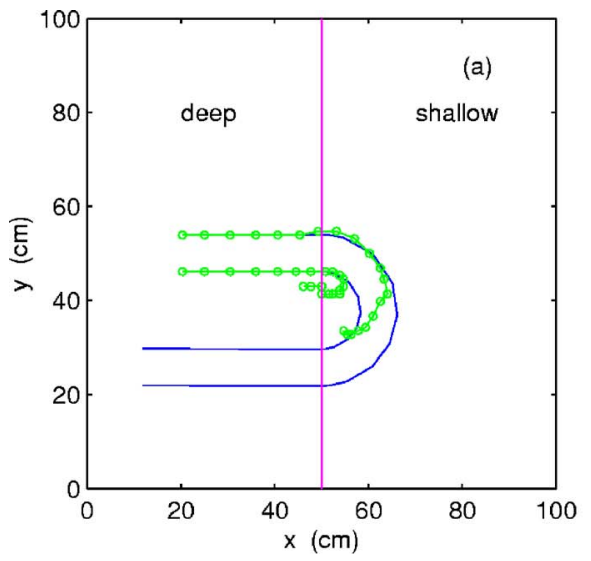

$$
\frac{U^{+}}{R+r}=\frac{U^{-}}{R},
$$

where $R$ is the radius of the inner circle. Therefore, this radius is given by

$$
R=\frac{U^{-} r}{U^{+}-U^{-}} .
$$

Using expressions (12) and (13), the calculated radius of the anticyclonic point vortex is $R=7.3 \mathrm{~cm}$. The parameters used are $f=1 \mathrm{~s}^{-1}, H_{0}=20 \mathrm{~cm}$, and $A_{0}=\pi r^{2}$, with $r=8 \mathrm{~cm}$. As can be seen in Fig. 9(a), the trajectories obtained with point vortices and with the barotropic model are very similar for about $120 \mathrm{~s}$, that is, about ten rotation periods of the system. For later stages, the trajectories do not coincide due to the viscous decay of the barotropic dipole. Note also that the point vortex dipole recovers the rectilinear trajectory as it returns to the deep region. Because the point vortex model is inviscid, the differences between the two models were to be expected. It is important to emphasize, however, that the deflection of the dipole trajectory is due mainly to inviscid, topographic effects. Repeating the same analysis for the case when the dipole starts at the shallow side [Fig. 9(b)], the calculated radius of the intensified cyclone is $R=8 \mathrm{~cm}$.

It can be concluded that the point vortex model represents well the modification of the dipole trajectory when it crosses the step, which is due to the change of circulation by squeezing and stretching effects. However, this model does not fully reproduce the dipole trajectories obtained with a continuous vorticity distribution formulation. The main reason is that viscous and nonlinear effects are absent in the point vortex model, which induce the decay an deformation of barotropic dipoles. Another reason for the difference between the model results and the experiment is the fact that the model does not include effects associated with vorticity generated near the step.

\section{DIPOLE ENCOUNTERING A HIGH STEP}

In this section, the interaction of a dipole with a step-like topography that has a significant height $(5 \mathrm{~cm})$ compared with the water depth $(20 \mathrm{~cm})$ is investigated. In contrast to the low-step case, the dipole is not able to cross the topography and it is sensibly distorted.

\section{A. Laboratory experiments}

Figure 10 shows a sequence of photographs of a typical qualitative experiment where the dipole starts at the deep region of the domain (left to right). In contrast with the $1 \mathrm{~cm}$ step, the vortex evolution is completely different: the dipole does not cross the step. Initially, the dipolar structure has a normal trajectory towards the step $(t=30-60 \mathrm{~s})$. As the vortex approaches the topographic feature, both structures move away from each other $(t=90)$ : the anticyclone moves back into the deep region, while the cyclone is distorted along the topography, losing coherence. It can be observed that part of the dye associated with the cyclone crosses towards the shallow region while the anticyclone is fully reflected from the step.

The reflection of the anticyclone approaching the step-up can be explained by means of a sequence of vorticity contours from a typical experiment, as shown in Fig. 11. This behavior is due to the influence of a cyclonic patch generated at the step $(t=30-60 \mathrm{~s})$, next to the anticyclone: this structure is formed by the counter-flow from shallow to deep water, which induces the creation of a cell of positive relative vorticity by stretching effects. This mechanism was analyzed by Zavala Sansón et al. ${ }^{10}$ for the reflection of monopolar
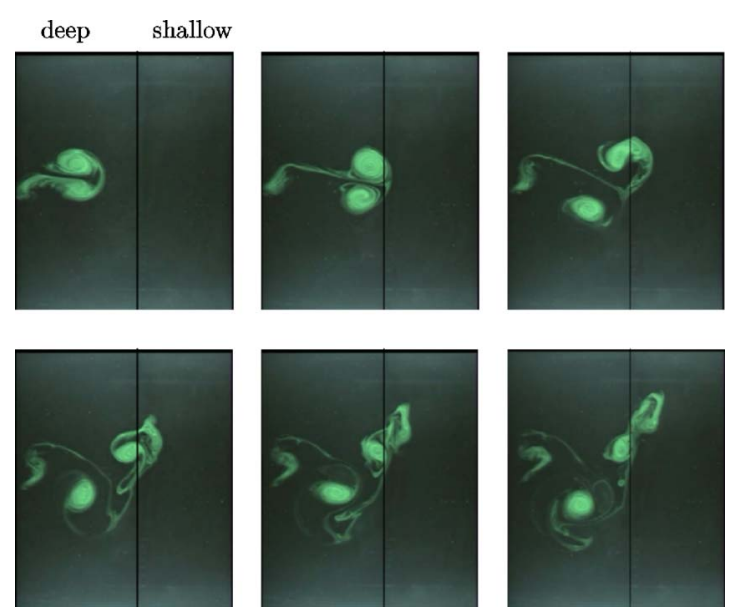

FIG. 10. Top view photographs showing the evolution of an experimental dipole crossing a high step $(5 \mathrm{~cm})$ from deep to shallow water during a timespan of $\sim 18$ rotation periods (time interval is $30 \mathrm{~s}$ ). The vortex is visualized with dye inside an area of $\sim 90 \mathrm{~cm} \times 100 \mathrm{~cm}$. 

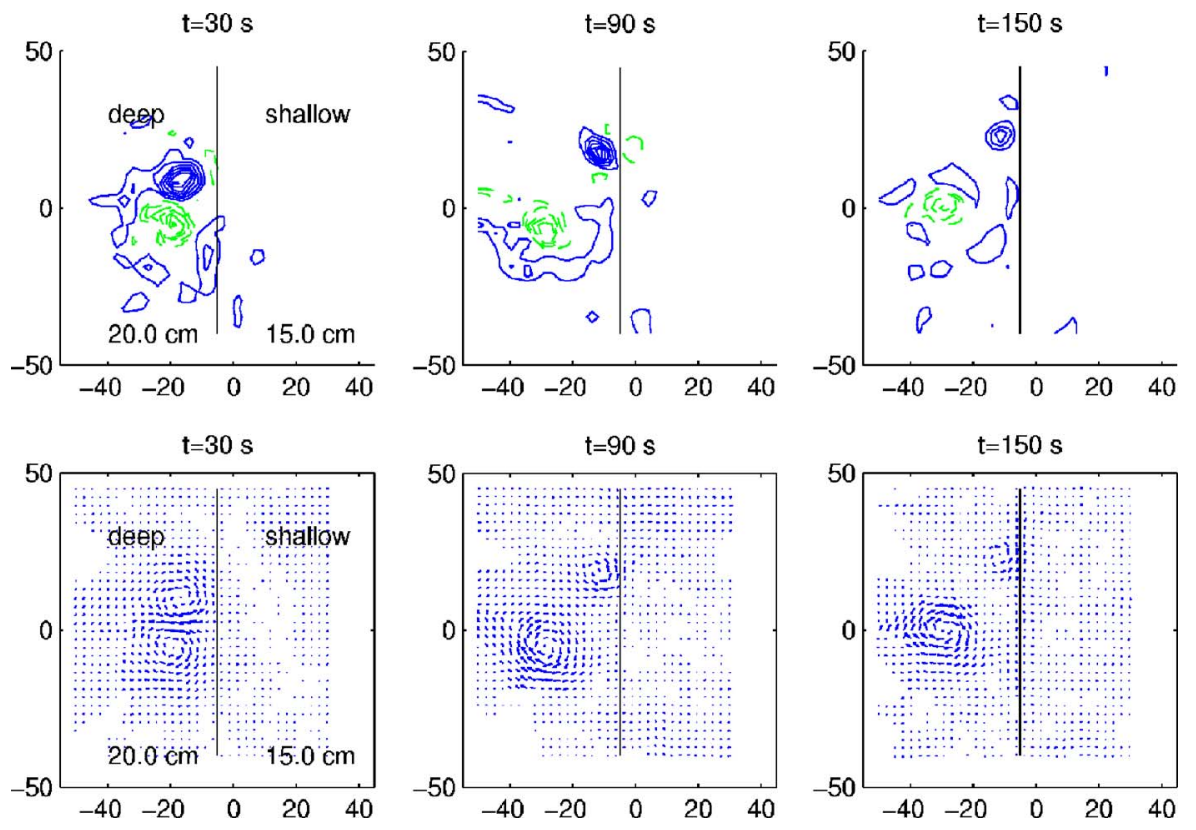

FIG. 11. Vorticity contours (upper row) and velocity field (lower row) of a dipole crossing a high step from deep to shallow water (as in previous figure) in a quantitative experiment. Black (gray) contours indicate positive (negative) vorticity values. The contour interval is $0.01 \mathrm{~s}^{-1}$, and the maximum/minimum vorticity is $1 /-1 \mathrm{~s}^{-1}$. vortices from a step-like topography. Since the cyclonic vorticity cell originated at the step is less intense, the new dipolar structure moving backwards has a curved trajectory in anticyclonic direction. On the other hand, the cyclonic part from the original structure slowly drifts along the step, towards the upper boundary. This motion is associated with a weak negative vorticity cell at the shallow side of the step (visible at $t=90 \mathrm{~s}$ ), generated by flow from deep to shallow water.

Figure 12 shows the qualitative behavior of a dipole that reaches the step from the shallow region. As in previous case, the cyclone and the anticyclone move away from each other as they approach the topography. As expected, the cyclonic part of the dipole is reflected from the step-down, analogously to the reflection of an anticyclone from a step-up. ${ }^{10}$ However, the anticyclonic structure seems to be
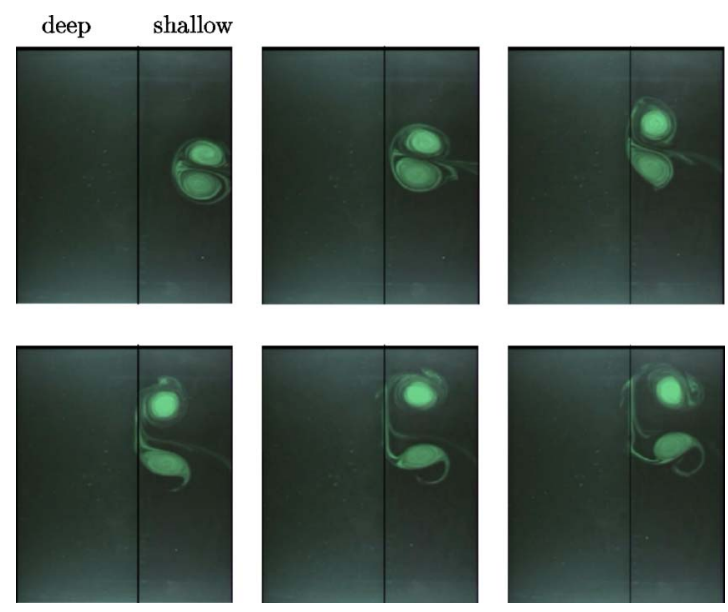

FIG. 12. Top view photographs showing the evolution of an experimental dipole crossing a high step $(5 \mathrm{~cm})$ from shallow to deep water during a timespan of $\sim 18$ rotation periods (time interval is $30 \mathrm{~s}$ ). The vortex is visualized with dye inside an area of $\sim 90 \mathrm{~cm} \times 100 \mathrm{~cm}$. reflected from the step too. The whole process occurs in a similar fashion as a dipole colliding with a wall. ${ }^{2}$

As mentioned above, the reflection of the cyclone from the step-down is clearly induced by topographic effects. This is shown in Fig. 13 presenting the corresponding evolution of vorticity contours from a quantitative experiment. It can be observed that the cyclone is reflected due to the interaction with a negative vorticity cell originated at the south of its position $(t=90 \mathrm{~s})$. This negative vorticity is created by the counter-flow from deep to shallow water. The behavior of the anticyclone is related with the formation of cyclonic vorticity along the step, distributed at the deep region $(t=30-150 \mathrm{~s})$. Apparently, this distribution gives rise to the anticyclone drift towards the upper part of the figure. For subsequent times, the structure slightly separates from the step, probably because of the interaction with the upper wall.

\section{B. Numerical simulations}

Figure 14 (upper panels) shows the sequence of vorticity contours from the simulation where the dipole approaches the step from deep to shallow water. As in the $1 \mathrm{~cm}$ step case, the numerical scheme reproduces the laboratory results very well. One observes the reflection of the anticyclone due to the formation of a positive vorticity patch $(t=30-150 \mathrm{~s})$, with the resultant dipolar structure moving backwards. It can be noticed that the cyclonic vorticity is rolled up around the anticyclone $(t=150-210 \mathrm{~s})$. This behavior captures quite well the vorticity measurements in Fig. 11. Note also that the original cyclonic part of the dipole is distorted and redistributed along the step ( $t=90-210 \mathrm{~s})$.

It is important to mark the behavior of passive tracers in Fig. 14 (lower panels), which can be compared with the dye experiment in Fig. 10. It can be seen that some particles initially inside the dipole atmosphere cross the topography $(t=90 \mathrm{~s})$, as in the qualitative experiments, where some dye associated with the cyclone crossed to the shallow part. The 

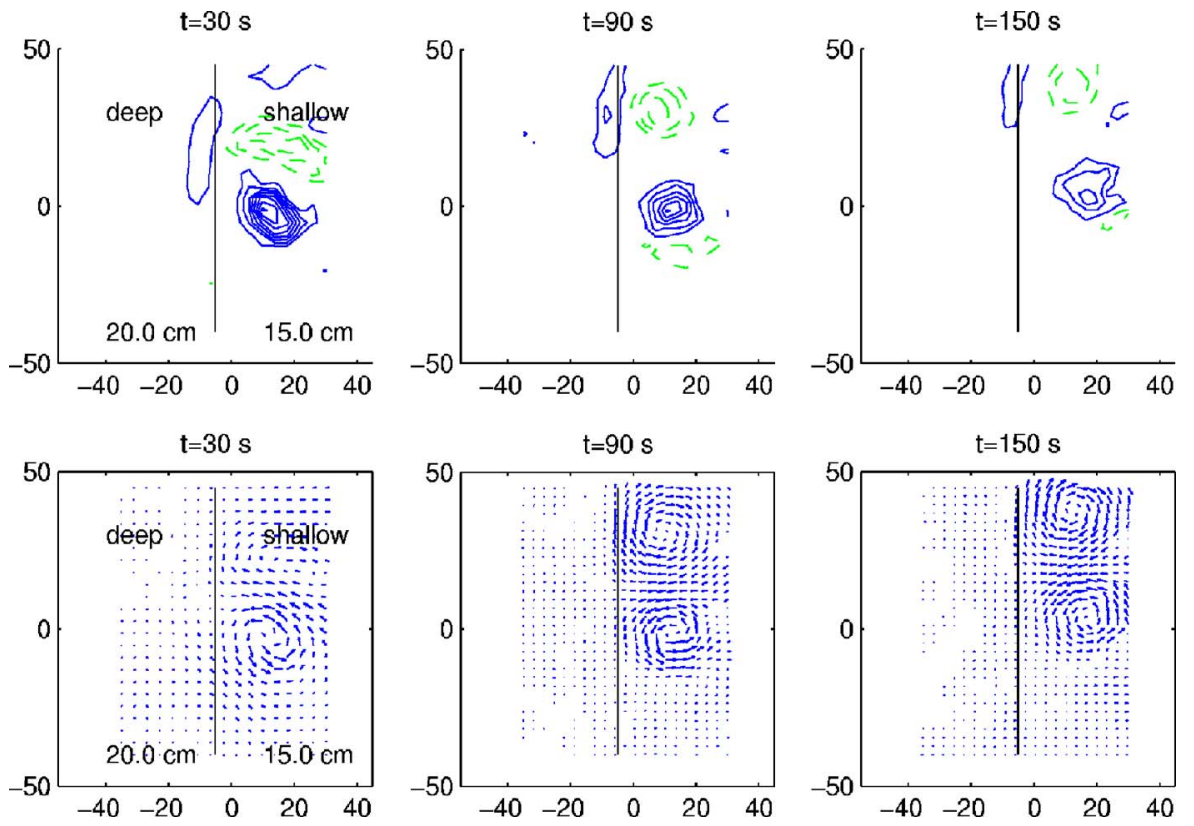

FIG. 13. Vorticity contours (upper row) and velocity field (lower row) of a dipole crossing a high step from shallow to deep water (as in previous figure) in a quantitative experiment. Vorticity contours as in Fig. 11. mechanism responsible for the reflection of the anticyclone is clear again: several particles initially distributed at the shallow region cross the step towards the deep region $(t=90 \mathrm{~s})$ acquiring positive relative vorticity, and inducing the backward drift of the anticyclone.

For the case in which the dipole reaches the step from the shallow side, some significant coincidences and differences between the experiments and the simulations are observed. Basically, the cyclone reflection is well represented by the simulation, while the anticyclone behavior is relatively different from the experiment. In Fig. 15 (upper panels) a sequence of vorticity contours is shown. The vorticity distribution represents the evolution of the cyclonic part of the dipole quite well, which is forced to move back towards the shallow region. In contrast, the anticyclonic part loses its structure and spreads along the topography. Although the reasons for the differences between this simulation and the experiment are not clear, it must be taken into account that the dipole's initial drift speed, its quasiperpendicular trajectory to the step, and the dipole dimensions on the laboratory experiments, might influence the final results. However, the cyclone reflection and the flow towards the upper wall along the step are well represented. Figure 15 (lower panels) shows the corresponding tracer evolution.

\section{CRITICAL STEP}

Although essentially different, the main characteristics of the flow behavior for low and high steps can be explained by using arguments of potential vorticity conservation (due to the weakness of viscous effects). Hence, a natural question arises: What is the critical step height under which dipoles are able to cross a step, and above which dipoles cannot cross? The process depends on many factors, such as the dipole dimensions, speed, circulation, and incidence angle. Here the step height and the dipole intensity are investigated with the help of the modulated point vortex model described in Sec. III.

Consider the case of a dipolar vortex encountering a step-up topography. When reaching the step, the anticylonic part of the dipole is under the influence of two cyclonic structures: the original positive part of the dipole, and the
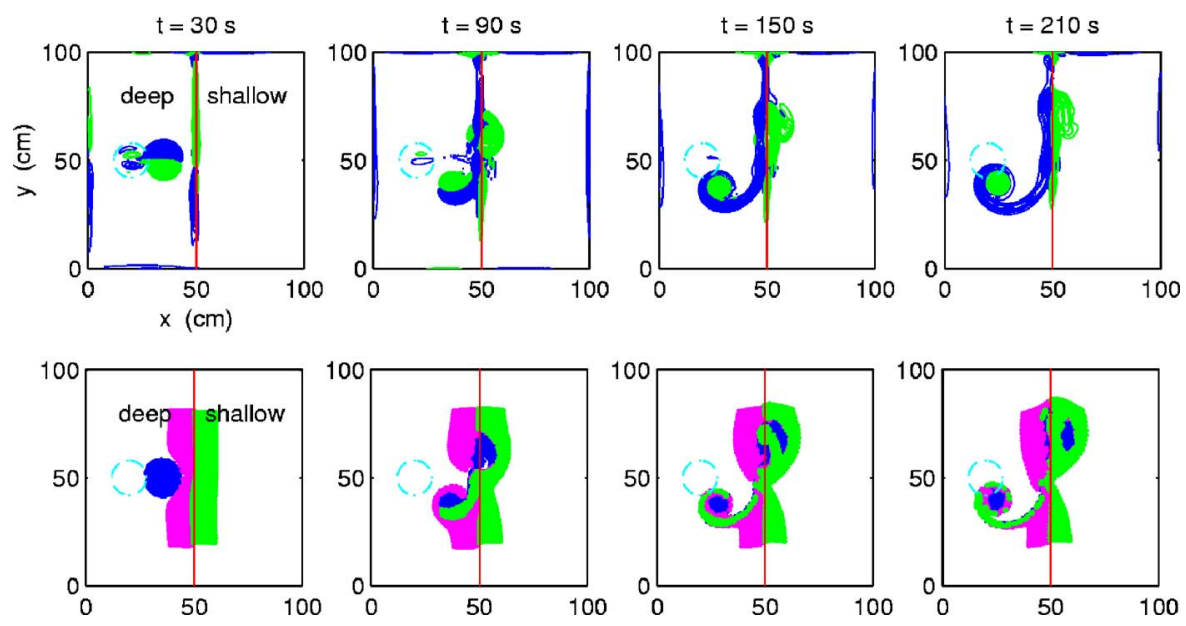

FIG. 14. Upper row: Numerically calculated vorticity contours of a dipole crossing a high step $(5 \mathrm{~cm})$ from deep to shallow water. Lower row: Evolution of passive tracers. Vorticity contours, dipole parameters, and initial arrangement of tracers as in Fig. 5. 

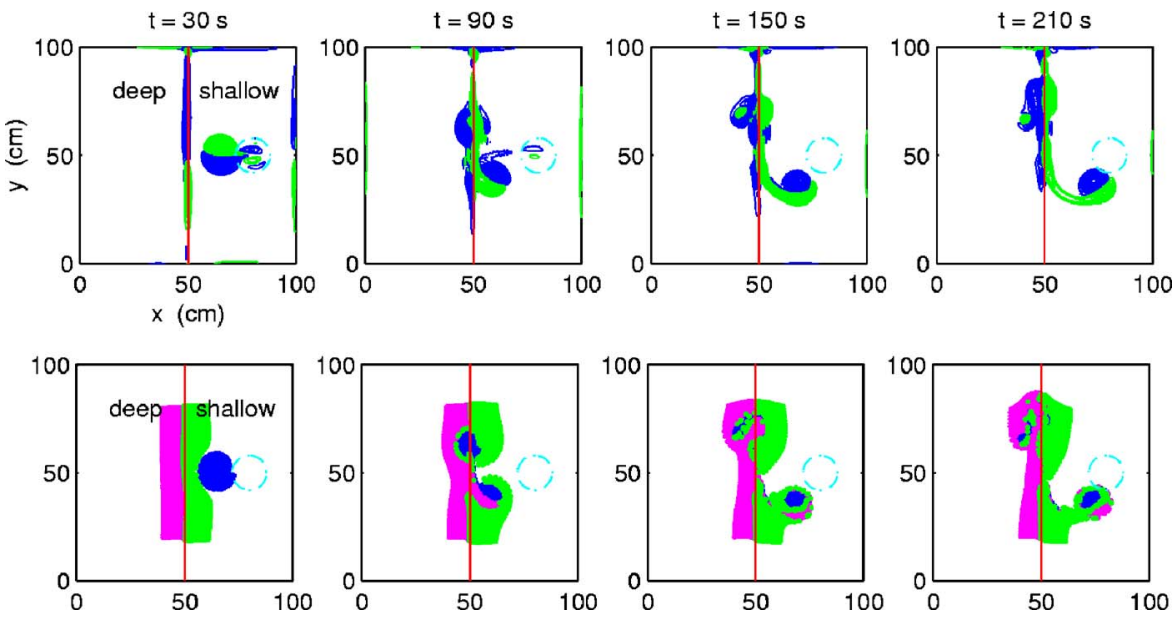

FIG. 15. Upper row: Vorticity contours of a dipole crossing a high step $(5 \mathrm{~cm})$ from shallow to deep water. Lower row: Evolution of passive tracers. Vorticity contours, dipole parameters and initial arrangement of tracers as in Fig. 5. topographically created patch of positive vorticity at the step (see, for instance, the vorticity contours at $t=90 \mathrm{~s}$ in Figs. 5 and 14). Their circulation is given by $\Gamma_{0}$ and, according to potential vorticity conservation, $f \alpha A_{0} \Delta h / H_{0}$, respectively. Here, $\Delta h>0$ is the step height, and $A_{0}=\pi a^{2} / 2$ the initial area of the positive side of the dipole. Note that the associated area of the topographically induced patch (which arises from fluid moving from shallow to deep water) is $\alpha A_{0}$, with $\alpha>0$. Therefore, the flow behavior can be predetermined by comparing the circulation of these two structures: if the cyclonic part of the dipole is more intense than the induced patch at the step, then the vortex crosses the topography; otherwise, the anticyclonic part is reflected. In nondimensional terms,

$$
\begin{aligned}
& \frac{\Gamma_{0}}{f A_{0}}>\alpha \frac{\Delta h}{H_{0}} \text { the whole dipole crosses the step, } \\
& \frac{\Gamma_{0}}{f A_{0}}=\alpha \frac{\Delta h}{H_{0}} \quad \text { critical step, } \\
& \frac{\Gamma_{0}}{f A_{0}}<\alpha \frac{\Delta h}{H_{0}} \text { the anticyclonic part is reflected. }
\end{aligned}
$$

Evidently, the right-hand side is equivalent to the Rossby number Ro. This relation indicates that knowing the original dipole characteristics $\left(\Gamma_{0}, A_{0}\right)$, it is possible to derive the relative step height $\left(\delta=\Delta h / H_{0}\right)$, for which the anticyclone crosses the topography due to the influence of the original cyclonic part, or it is reflected back into the deep region. Note that $\alpha^{-1}=\delta / R o$ is the same parameter used by Dunn et al. ${ }^{11}$

In order to analyze how these results can be used to predict whether a dipolar vortex is able to cross a step topography or not, the critical curve $\Gamma_{0} / f A_{0}=\alpha \Delta h / H_{0}$ is plotted in Fig. 16. The number $\alpha$ is chosen to divide the parameter space in regions of dipole crossing (below the curve) and reflection (above the curve). This is achieved by performing several numerical simulations using different steps and initial dipole circulations, indicated in the same figure. Note that the dipole parameters can be written in terms of the initial speed and radius of the atmosphere by taking $\Gamma_{0} \approx 6.83 a U_{0} \cdot{ }^{17}$ The criterion to determine that a dipole passed the step consisted of verifying that the peak vorticity of the cyclonic and anticyclonic parts crossed the topography. Thus, by choosing $\alpha=3.7$ it is found that simulations with flow parameters above the continuous line, i.e., high steps or weak dipoles, are cases in which the vortex is not able to cross the step and the anticyclonic structure is reflected. On the contrary, for low steps or intense dipoles, vortices do cross the topography with a deflected trajectory. Cases A and B in Fig. 16 are clearly representative of the respective situations, as shown by a snapshot of their calculated vorticity contours. This trend is also very clear for other cases well above or below the curve. Simulations near the critical step are expected to show a somewhat ambiguous behavior. This can be appreciated from a snapshot of vorticity contours in cases $\mathrm{C}$ and $\mathrm{D}$, whose parameters are very close to both sides of the curve. Nevertheless, the predicted behavior is also observed. It is important to point out that the barotropic simulations included only lateral viscous effects and no Ekman friction, while Eq. (16) is derived from purely inviscid arguments. Despite this, the prediction for the dipole behavior is rather correct.

\section{SUMMARY}

The interaction of a dipolar vortex with a step-like topography in a rotating system has been investigated by means of laboratory experiments and numerical simulations. In order to understand the influence of the topography, two step heights were used, i.e., $\Delta h=1$ and $5 \mathrm{~cm}$, with a $H_{0}=20 \mathrm{~cm}$ maximum depth. These configurations have been called low and high steps, respectively, since the observed results on the evolution of weak (small Rossby number) dipolar vortices depend dramatically on the step height.

For the low-step case $\left(\Delta h / H_{0}=0.05\right)$, the vortex crosses the topography with a deflected trajectory, while maintaining its dipolar structure. The sense of this deflection depends on whether the dipole reaches a step-up or a step-down: when crossing a step-up (down) the trajectory is deviated towards the right (left); i.e., in anticyclonic (cyclonic) direction. This deflection is clearly explained by potential vorticity conservation: when the dipole crosses a step-up, its anticyclonic part is intensified due to squeezing effects, while the cyclonic part is weakened. As a result, the dipole trajectory is de- 


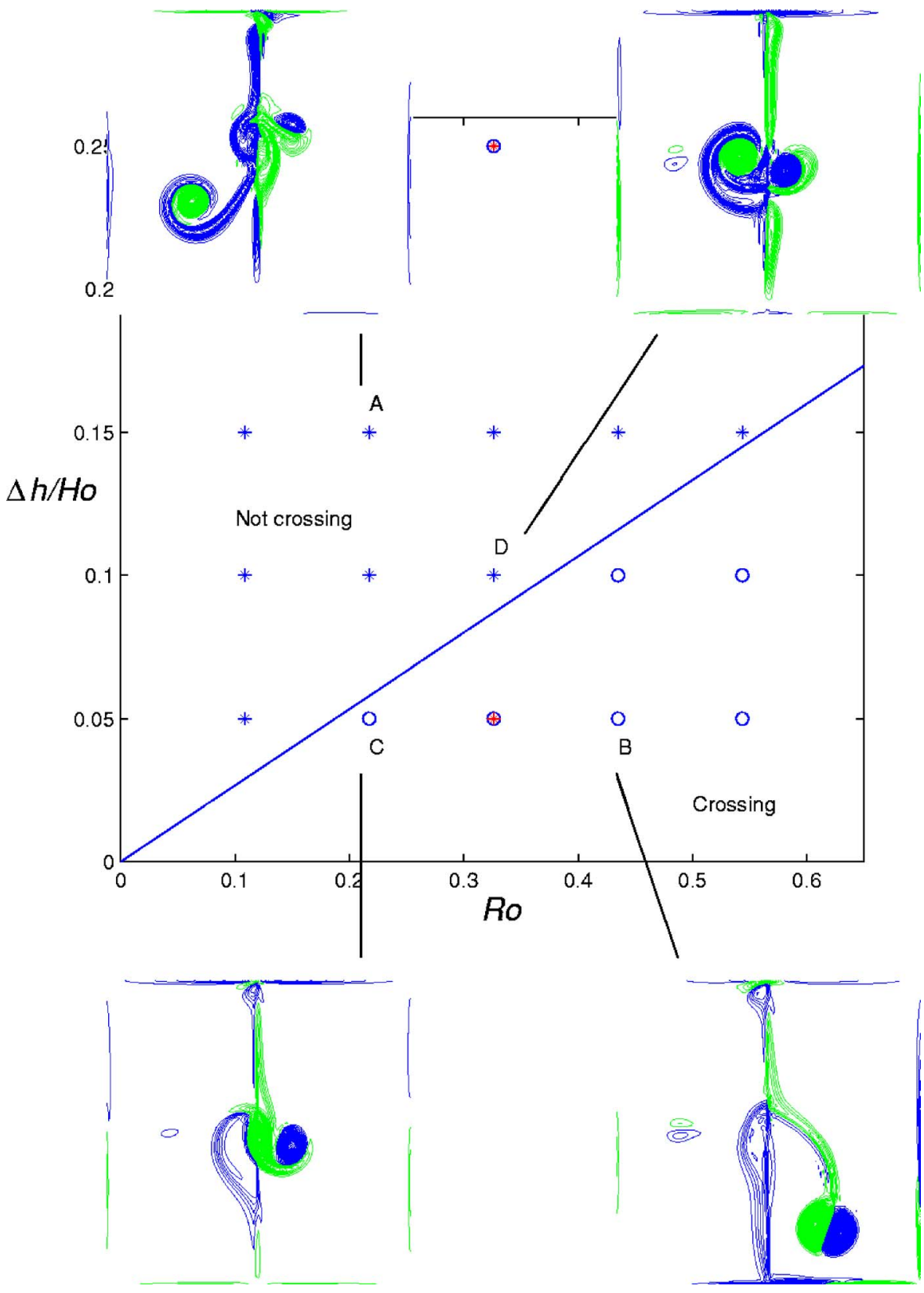

FIG. 16. Parameter space defined by the relative step-up height, $\Delta h / H_{0}$, and the Rossby number, $\mathrm{Ro}=\Gamma_{0} / f A_{0}$. The solid line indicates the critical relative step according to (16) using $\alpha=3.75$. Stars (circles) above (below) the curve denote simulations where dipoles do not cross (do cross) the step. Typical cases of both situations are shown by snapshots of cases A and D. Two cases near both sides of the critical curve are also shown (B and C). flected towards the more intense part; that is, the anticyclone. Equivalent arguments apply when the dipole encounters a step-down. Another relevant result is the formation of a weak flow along the step with shallow water on its right.

For the high-step $\left(\Delta h / H_{0}=0.25\right)$, in contrast, the dipole is not able to cross the topography. In this case, the reflection of one of the dipole structures is observed: the anticyclone (cyclone) if the dipole reaches the step from the deep (shallow) region. For the first case, for instance, a positive relative vorticity cell arises due to the flow from shallow to deep water, as the dipole approaches the step. This new structure pairs with the anticyclonic part of the dipole, preventing its motion towards the shallow region. This reflection mechanism was discussed by Zavala Sansón et al. ${ }^{10}$ for monopolar vortices drifting on a topographic $\beta$ plane towards a step-like topography. Moreover, in a similar fashion as in the low step case, a weak flow along the topography maintaining the shallow region on its right is generated.
The symmetry of the dipole evolution in the presence of a step-up or a step-down deserves an additional remark. It is evident that the barotropic model (1) is not invariant under transformations

$$
T:(\psi, \Delta h) \rightarrow(-\psi,-\Delta h)
$$

However, the numerical results are nearly symmetrical under this transformation, that is, the evolution of cyclonic vorticity when the dipole approaches a step-up is very similar to the evolution of anticyclonic vorticity when approaching a step-down (see, e.g., Figs. 5 and 6). Evidently, this likeness is due to the relatively low value of the ratio $\Delta h / H_{0}$, even for the $5 \mathrm{~cm}$ step. By writing the fluid depth $h=H_{0}\left(1-\Delta h / H_{0}\right)$ and assuming $\Delta h / H_{0}<<1$, the vorticity equation can be written as [neglecting terms $O\left(\omega \Delta h, \Delta h^{2}\right)$ ], 


$$
\frac{\partial \omega}{\partial t}+J\left(q_{G}, \psi^{*}\right)=\nu \nabla^{2} \omega-\frac{1}{2} E^{1 / 2} f \omega,
$$

where the quasigeostrophic potential vorticity is given by $q_{G}=\omega+f \Delta h / H_{0}{ }^{4}$ The only remaining bottom friction effect is provided by the well-known linear Ekman term. Note that this vorticity equation, is invariant under transformation (17), which explains the observed symmetry in simulations and experiments (similar arguments were used by Zavala Sansón et al., ${ }^{10}$ including the $\beta$ effect).

\section{ACKNOWLEDGMENTS}

The experimental results were obtained at the Fluid Dynamics Laboratory of Eindhoven University of Technology. We gratefully acknowledge the help of Karen Mullener with some of the experiments.

${ }^{1}$ E. J. Hopfinger and G. J. F. van Heijst, "Vortices in rotating fluids," Annu. Rev. Fluid Mech. 25, 241 (1993).

${ }^{2}$ P. Orlandi, "Vortex dipole rebound from a wall," Phys. Fluids A 2, 1429 (1990).

${ }^{3}$ G. F. Carnevale, O. U. Velasco Fuentes, and P. Orlandi, "Inviscid dipolevortex rebound from a wall or coast," J. Fluid Mech. 351, 75 (1997).

${ }^{4}$ L. Zavala Sansón and G. J. F. van Heijst, "Ekman effects in a rotating flow over bottom topography," J. Fluid Mech. 471, 239 (2002).
${ }^{5}$ Y. H. Spitz and D. Nof, "Separation of boundary currents due to bottom topography," Deep-Sea Res., Part A 38, 1 (1991).

${ }^{6}$ M. E. Stern and J. Austin, "Entrainment of shelf water by a bifurcating continental boundary current," J. Phys. Oceanogr. 25, 3118 (1995).

${ }^{7}$ L. Zavala Sansón, R. Serravall, G. F. Carnevale, and G. J. F. van Heijst, "Experiments and simulations on coastal flows in the presence of a topographic slope,” J. Phys. Oceanogr. 35, 2204 (2005).

${ }^{8}$ B. W. An and N. R. McDonald, "Coastal currents and eddies and their interaction with topography," Dyn. Atmos. Oceans 40, 237 (2005).

${ }^{9}$ G. F. Carnevale, S. G. L. Smith, F. Crisciani, R. Purini, and R. Serravall, "Bifurcation of a coastal current at an escarpment," J. Phys. Oceanogr. 29, 969 (1999)

${ }^{10}$ L. Zavala Sansón, G. J. F. van Heijst, and J. J. J. Doorschot, "Reflection of barotropic vortices from a step-like topography," Nuovo Cimento Soc. Ital. Fis., C 22, 909 (1999).

${ }^{11}$ D. C. Dunn, N. R. McDonald, and E. R. Johnson, "The motion of a singular vortex near an escarpment," J. Fluid Mech. 448, 335 (2001).

${ }^{12}$ D. C. Dunn, "The evolution of an initially circular vortex near an escarpment. Part II: numerical results," Eur. J. Mech. B/Fluids 21, 677 (2002).

${ }^{13}$ L. Zavala Sansón, G. J. F. van Heijst, and N. A. Backx, "Ekman decay of a dipolar vortex in a rotating fluid," Phys. Fluids 13, 440 (2001).

${ }^{14}$ O. U. Velasco Fuentes and G. J. F. van Heijst, "Experimental study of dipolar vortices on a topographic $\beta$-plane," J. Fluid Mech. 259, 79 (1994).

${ }^{15}$ V. V. Meleshko and G. J. F. van Heijst, "On Chaplygin's investigations of two-dimensional vortex structures in an inviscid fluid," J. Fluid Mech. 272, 157 (1994)

${ }^{16}$ A. H. Nielsen and J. Juul Rasmussen, "Formation and temporal evolution of the Lamb-dipole," Phys. Fluids 9, 982 (1997).

${ }^{17}$ J. B. Flór, G. J. F. van Heijst, and R. Delfos, "Decay of dipolar vortex structures in a stratified fluid," Phys. Fluids 7, 374 (1995). 